\title{
ON THE BOUNDEDNESS AND UNBOUNDEDNESS OF CERTAIN CONVOLUTION OPERATORS ON NILPOTENT LIE GROUPS
}

\author{
ROE GOODMAN
}

\begin{abstract}
One method of proving irreducibility of the "principal series" representations of semisimple Lie groups involves showing that a certain nonintegrable function on a nilpotent subgroup $X$ cannot be regularized to give a bounded convolution operator on $L_{2}(X)$. This note gives an elementary proof of this unboundedness property for the groups $X$ which occur in real-rank one semisimple groups.
\end{abstract}

Introduction. Let $X$ be a connected, simply-connected nilpotent Lie group with a one-parameter group $\left\{\delta_{r} ; 0<r<\infty\right\}$ of dilations and a norm function $|x|$, as defined by Knapp and Stein in [2]. For example, $X=\boldsymbol{R}^{n}$ with $\delta_{r} x=r x$ (scalar multiplication), and $|x|=\|x\|^{n}$, where $\|\cdot\|$ is the Euclidean norm. Our purpose in this note is to obtain some elementary results on the boundedness and unboundedness of regularizations of the nonintegrable function $|x|^{-1}$, acting by convolution on various function spaces. In particular, we obtain an independent proof of the "unboundedness theorem" of [2], i.e. the fact that no distribution obtained by regularization of $|x|^{-1}$ at the identity is a bounded convolution operator on $L_{2}(X)$. Our approach (suggested by the proof of the discrete version of this theorem in Hardy-Littlewood-Pólya [1, p. 214]) is to exploit the nonintegrability of $|x|^{-1}$ at infinity. This requires control over the singularity of $|x|^{-1}$ at the identity, which we gain by proving a simple "boundedness" theorem in $\S 1$. The general unboundedness theorem of Knapp and Stein is an easy corollary of the unboundedness of the "minimal" regularization of $|x|^{-1}$, which we establish in $\S 2$.

1. Boundedness theorem. Before stating the theorem, we recall some properties of the norm function $|x|$ (cf. [2, §§2 and 5]). We have $x \rightarrow|x|$ continuous on $X$ and $C^{\infty}$ on $X-\{e\}$ (we use $e$ to denote the identity element of $X)$, with $\left|x^{-1}\right|=|x|$ and $|x|>0$ if $x \neq e$. Under dilations it transforms by $\left|\delta_{r} x\right|=r^{q}|x|$, where $q>0$. If $d x$ denotes a Haar measure on $X$, then

Received by the editors May 16, 1972.

AMS (MOS) subject classifications (1970). Primary 22E30, 44A25.

c) American Mathematical Society 1973 
$d\left(\delta_{r} x\right)=r^{a} d x$, so that $|x|^{-1} d x$ is invariant under $\left\{\delta_{r}\right\}$. The following inequalities hold:

$$
\begin{gathered}
|x y| \leqq A \max \{|x|,|y|\}, \\
|| x y|-| y|| \leqq C_{0}|x|^{d}|y|^{1-d} \quad \text { if }|x| \leqq|y| .
\end{gathered}
$$

Here $A>1$, while $0<d \leqq 1$. (If $\left\{p_{i}\right\}$ are the eigenvalues of the generator $D$ of $\left\{\delta_{r}\right\}$, then $q=\sum p_{i}$ and $d=\min \left\{p_{i}\right\} \mid q$.) We also need the following integration formula: If $f(x)=\phi(|x|)$, where $\phi$ is a nonnegative Borel function on $R^{+}=(0, \infty)$, then

$$
\int_{X} f(x) d x=\int_{R^{+}} \phi(t) d t,
$$

provided we normalize the Haar measure $d x$ so that $\int_{1 \leqq|x| \leqq e}|x|^{-1} d x=1$.

THEOREM 1. Let $\sigma$ be the distribution

$$
\sigma(f)=\int_{|x| \leqq 1}\{f(x)-f(e)\}|x|^{-1} d x,
$$

$f \in C_{0}^{\infty}(X)$. Let $\mathscr{R}$ be the subspace of $C_{0}^{\infty}(X)$ consisting of all $f$ of the form $f(x)=\phi(|x|)$, where $\phi \in C_{0}^{\infty}\left(R^{+}\right)$. For such a function $f$, define

$$
N(f)=\left\{\int_{0}^{\infty}\left|\phi^{\prime}(t)\right|^{2}\left(1+t^{2-d}\right) d t\right\}^{1 / 2}
$$

( $d$ as in inequality (2)). Then there exists a constant $\beta>0$ so that

$$
\|\sigma * f\|_{2} \leqq \beta N(f)
$$

for all $f \in \mathscr{R}$.

Proof. Define $\left(L_{x} f\right)(y)=f\left(x^{-1} y\right)$. Then by Schwarz' inequality,

$$
\|\sigma * f\|_{2}^{2} \leqq C_{\varepsilon} \int_{|x| \leqq 1}\left\|L_{x} f-f\right\|_{2}^{2} \frac{d x}{|x|^{1+\varepsilon}},
$$

where $C_{\varepsilon}=\int_{|x| \leqq 1}|x|^{-1+\varepsilon} d x$, and $\varepsilon>0$. Suppose that $f \in \mathscr{R}, f(x)=\phi(|x|)$. Using the integration formula (3), to represent $f$ as the integral of $\phi^{\prime}$, and applying Schwarz' inequality again, we get the bound

$$
\left|f\left(x^{-1} y\right)-f(y)\right|^{2} \leqq|| x^{-1} y|-| y|| \int_{\left[\left|x^{-1} y\right|,|y|\right]}\left|\phi^{\prime}(t)\right|^{2} d t .
$$

We will integrate (7) to obtain an estimate for the integrand in (6). Consider first the region $|y| \geqq A|x|$ (the constant $A$ as in (1)). In this 
region $\left[\left|x^{-1} y\right|,|y|\right] \subseteq\left[A^{-1}|y|, A|y|\right]$ and (2) holds. Hence

$$
\begin{aligned}
\int_{|y| \geqq A|x|}\left|f\left(x^{-1} y\right)-f(y)\right|^{2} d y & \leqq C_{0}|x|^{d} \int_{|y| \geqq A|x|}\left\{\int_{A^{-1}|y|}^{A|y|}\left|\phi^{\prime}(t)\right|^{2} d t\right\}|y|^{1-d} d y \\
& \leqq C_{1}|x|^{d} \int_{0}^{\infty}\left|\phi^{\prime}(t)\right|^{2} t^{2-d} d t,
\end{aligned}
$$

where we have interchanged the order of integration and used formula (3) again, and the constant $C_{1}$ is independent of $\phi$. In the region $|y| \leqq A|x|$ we simply use the estimate $\left|x^{-1} y\right| \leqq A^{2}|x|$ to obtain from (7) the bound

$$
\int_{|y| \leqq A|x|}\left|f\left(x^{-1} y\right)-f(y)\right|^{2} d y \leqq 2 A^{2}|x| \int_{0}^{\infty}\left|\phi^{\prime}(t)\right|^{2} d t .
$$

Assume now that $|x| \leqq 1$. Then $|x| \leqq|x|^{d}$, since $d \leqq 1$, so by the estimates just made,

$$
\left\|L_{x} f-f\right\|_{2}^{2} \leqq C_{2}|x|^{d} \int_{0}^{\infty}\left|\phi^{\prime}(t)\right|^{2}\left(1+t^{2-d}\right) d t,
$$

$C_{2}$ a constant independent of $\phi$. If we take $\varepsilon=d / 2$ in (6) and use estimate (8), we obtain (5). Q.E.D.

\section{Unboundedness theorem.}

THEOREM 2. Let $T$ be the distribution

$$
T(f)=\sigma(f)+\int_{|x| \geqq 1} f(x)|x|^{-1} d x
$$

( $\sigma$ as in Theorem 1). Then left convolution by $T$ is not bounded on $L_{2}(X)$.

Proof. If $v$ is the distribution

$$
v(f)=\int_{|x| \geqq 1} f(x)|x|^{-1} d x,
$$

then it is easy to show that convolution by $v$ is not bounded on $L_{2}$. Indeed, let $\left\{\phi_{n}\right\} \subseteq C_{0}^{\infty}\left(R^{+}\right)$be a sequence of nonnegative functions such that $\phi_{n}(t)=t^{-1 / 2}(\log t)^{-1}$ in the interval $e \leqq t \leqq A^{2} e^{n}$, with $\phi_{n}$ smoothly cut off outside this interval in such a way that $\sup _{n} \int_{0}^{\infty} \phi_{n}(t)^{2} d t<\infty$ (this is certainly possible, since $\left.\int_{e}^{\infty} t^{-1}(\log t)^{-2} d t<\infty\right)$. Set $f_{n}(x)=\phi_{n}(|x|)$. By formula (3), $\left\{f_{n}\right\}$ is a bounded sequence in the $L_{2}$ norm. On the other hand, when $e \leqq|x| \leqq e^{n}$, then

$$
\left(f_{n} * f_{n}\right)(x) \geqq \int_{A|x| \leqq|y| \leqq A e^{n}} f_{n}\left(x^{-1} y\right) f_{n}(y) d y
$$


But in the range $A|x| \leqq|y|, e \leqq|x| \leqq e^{n}$, one has also $e \leqq A^{-1}|y| \leqq\left|x^{-1} y\right| \leqq$ $A|y|$. Hence from the definition of $f_{n}$ we get the lower bound

$$
\begin{aligned}
\left(f_{n} * f_{n}\right)(x) & \geqq C_{1} \int_{A|x| \leqq|y| \leqq A e^{n}}|y|^{-1}(\log |y|)^{-2} d y \\
& \geqq C_{2}(\log |x|)^{-1} \quad \text { if }|x| \leqq e^{n / 2},
\end{aligned}
$$

where $C_{2}>0$ is independent of $n$.

Integrating this estimate in the range $e \leqq|x| \leqq e^{n / 2}$, we conclude that $v\left(f_{n} * f_{n}\right) \geqq C_{2} \log (n / 2)$, and hence convolution by $v$ is not bounded on $L_{2}$. (Remark. $\nu \in L_{p}$ for all $p>1$, so by Young's inequality convolution by $v$ is bounded from $L_{r}$ to $L_{2}$ for any $r<2$. The sequence $\left\{f_{n}\right\}$ was chosen to be bounded in $L_{2}$ but not bounded in $L_{r}$ for any $r<2$.)

To prove Theorem 2 , we only need to show that a sequence $\left\{f_{n}\right\}$ as above can be constructed with the additional property that $\sigma\left(f_{n} * f_{n}\right)$ remains bounded as $n \rightarrow \infty$. By Theorem 1 this will be the case provided that $\sup _{n} N\left(f_{n}\right)<\infty$. This can be easily done, however. The only delicate point is to truncate the functions $\phi_{n}$ slowly enough as $t \rightarrow \infty$ so that $\phi_{n}^{\prime}$ is sufficiently small (this keeps $N\left(f_{n}\right)$ bounded), but yet rapidly enough to keep $\left\|f_{n}\right\|_{2}$ bounded. Specifically, we construct $\phi_{n} \in C_{0}^{\infty}\left(\boldsymbol{R}^{+}\right)$as above, $\phi_{n}(t)=t^{-1 / 2}(\log t)^{-1}$ in $e \leqq t \leqq A^{2} e^{n} \equiv a_{n}$, requiring that $\phi_{n}(t)=0$ for $t>\left(1+e^{-n d}\right) a_{n} \equiv b_{n}$, and taking a fixed cut-off in $1 \leqq t \leqq e$. In the "cut-off" interval $\left[a_{n}, b_{n}\right]$ we require

$$
0 \leqq \phi_{n}(t) \leqq M n^{-1} e^{-n / 2}, \quad\left|\phi_{n}^{\prime}(t)\right| \leqq M \exp [n(-3 / 2+d)],
$$

where $M$ is a fixed constant. This can certainly be done, since we can construct a $C^{1}$ function $\psi_{n}$ with these properties (the estimate for the derivative being obtained by comparison with a linear cut-off in $\left.\left[a_{n}, b_{n}\right]\right)$, and then set $\phi_{n}=\theta * \psi_{n}$, where $\theta \in C_{0}^{\infty}(R)$ is fixed.

Suppose the sequence $\left\{\phi_{n}\right\}$ has been constructed, and set $f_{n}(x)=$ $\phi_{n}(|x|)$. Then

$$
\left\|f_{n}\right\|_{2}^{2} \leqq C+\int_{e}^{a_{n}} t^{-1}(\log t)^{-2} d t+\left(b_{n}-a_{n}\right) M^{2} n^{-2} e^{-n} .
$$

But $b_{n}-a_{n}=A^{2} e^{(1-d) n}$, and $d>0$, so $\sup _{n}\left\|f_{n}\right\|_{2}<\infty$. Furthermore,

$$
\begin{aligned}
N\left(f_{n}\right)^{2} \leqq & C+\int_{e}^{a_{n}} \frac{2\left(1+t^{2-d}\right)}{t^{3}(\log t)^{2}} d t \\
& +\left(b_{n}-a_{n}\right)\left(1+b_{n}^{2-d}\right) \sup _{a_{n} \leqq t \leqq b_{n}}\left|\phi_{n}^{\prime}(t)\right|^{2} .
\end{aligned}
$$

By construction of $\phi_{n}$, the right side is bounded as $n \rightarrow \infty$, as required. 
Corollary (KNAPP AND Stein). Let $\mu$ be any distribution on $X$ such that $\mu=|x|^{-1}$ on $X \sim\{e\}$. Then convolution by $\mu$ is unbounded on $L_{2}(X)$.

Proof. Let $T$ be the distribution of Theorem 2. Then $\mu-T$ vanishes on $X-\{e\}$, and hence is a finite linear combination of derivatives of the point mass at $e$. Pick a fixed, nonnegative $\phi=\phi^{*} \in C_{0}^{\infty}(X)$, and set $\phi_{r}(x)=$ $(\log r)^{-1 / 2} r^{q / 2} \phi\left(\delta_{r} x\right)$. Since $d\left(\delta_{r} x\right)=r^{q} d x$, while $|x|^{-1} d x$ is invariant under $\delta_{r}$, we have

$$
\begin{aligned}
(\log r) T\left(\phi_{r} * \phi_{r}\right)= & \iint_{|x| \leqq r^{a}}[\phi(x y)-\phi(y)] \phi(y)|x|^{-1} d x d y \\
& +\iint_{|x|>r^{a}} \phi(y) \phi(x y)|x|^{-1} d x d y \\
= & T(\phi * \phi)-q(\log r)\|\phi\|_{2}^{2} .
\end{aligned}
$$

Also $\left\|\phi_{r}\right\|_{2}=(\log r)^{-1 / 2}\|\phi\|_{2}$. Hence $T\left(\phi_{r} * \phi_{r}\right)$ and $\left\|\phi_{r}\right\|_{2}$ are bounded as $r \rightarrow \infty$. On the other hand, if $\left\{X_{i}\right\}$ is an eigenvector for the differential of $\delta_{e}$, then $\left(\partial / \partial X_{i}\right) \phi_{r} * \phi_{r}(e)=(\log r)^{-1} r^{p_{i}}\left(\partial \phi / \partial X_{i}\right) * \phi(e)$. Since all $p_{i}>0$, it is clear that $\mu\left(\phi_{r} * \phi_{r}\right)$ cannot be bounded as $r \rightarrow \infty$ unless $\mu-T$ is a multiple of the identity operator. Hence the assumption that convolution by $\mu$ is bounded on $L_{2}$ leads to the conclusion that convolution by $T$ is bounded on $L_{2}$, contradicting Theorem 2. Q.E.D.

\section{REFERENCES}

1. G. H. Hardy, J. E. Littlewood and G. Pólya, Inequalities, 2nd ed., Cambridge Univ. Press, New York, 1952. MR 13, 727.

2. A. W. Knapp and E. M. Stein, Intertwining operators for semi-simple groups, Ann. of Math. (2) 93 (1971), 489-578.

Department of Mathematics, Rutgers University, New Brunswick, New Jersey 08903 